\title{
Short Term Outcomes of Lichtenstein Hernioplasty Versus Desarda Technique in Repair of Inguinal Hernia
}

\author{
Aya M. Selim ${ }^{\text {a }}$, Mohamed K. Elamary ${ }^{\text {, }}$ Mohamed Y. Ahmed ${ }^{\mathrm{a}}$, Mohammed A. Omar ${ }^{\mathrm{a}}$
}

${ }^{\mathrm{a}}$ General Surgery Department, Qena Faculty of Medicine, South Valley University.

\begin{abstract}
:
Background: The Desarda repair technique of inguinal hernia repair introduced in 2001 is still not considered standard tissue based hernia repair technique. We compared the tissue based Desarda technique with standard Lichtenstein repair in treatment of primary inguinal hernia.

Objectives: The aim of this study was to compare the short term outcomes of Desarda technique versus Lichtenstein hernioplasty in inguinal hernia repair.

Patients and methods: A total of 81 participants (41 in the Lichtenstein arm and 40 in the Desarda arm) were enrolled into this single centre double-blind randomised controlled trial. The outcome measures were evaluated at 1-2 h, 3, 7 and 14 days. The primary outcomes measured were recurrence and chronic pain. In addition to early and late complications, foreign body sensation, and return to everyday activity which examined and evaluated in hospital after surgery.

Results: During one year follow up, no recurrence was detected in each group . Chronic groin pain was experienced by $5.6 \%$ and $4.2 \%$ of patients from Desarda and Lichtenstein groups respectively $(\mathrm{P}=0.68)$. There was no significant statistical difference in mean postoperative VAS scores for pain at the five time points between the two study groups. There was significantly shorter operating time and earlier return to normal gait in favor of Desarda repair. Foreign body sensation was not different between the two groups..

Conclusions: Successful inguinal hernia treatment without mesh implantation can be achieved using Desarda repair, as it is effective as the standard Lichtenstein procedure. Shorter operating time, early return to normal gait and lower cost (no mesh) are potential benefits of Desarda repair. The suitability of Desarda repair for patients found to have thin, weak or divided external oblique aponeurosis intraoperatively needs further evaluation.
\end{abstract}

Keywords: Inguinal hernia, Desarda repair Tissue-based repair, Lichtenstein procedure, Mesh repair

\section{Introduction}

A hernia is an abnormal protrusion of a viscus or a part of viscus through an opening in the wall of cavity containing it. Because of their frequency, inguinal hernias remain an important medical problem. The estimated lifetime risk for inguinal hernia is $27 \%$ for men and 3\% for women (Primatesta; Goldacre 1996).

Annual morbidity rates in various countries vary from 100 to 300 per 100,000citizens(Bay-Nielsen et al., 2001).

The synthetic prostheses most often used in the inguinal area can cause foreign bodysensation in the groin, discomfort, and abdominal wall stiffness(D'Amore et al., 2008). Surgical-site infections are morefrequent after hernia treatment using $\operatorname{mesh}($ Genc et al., 2010). Migration of the mesh from the primary site of implantation in the abdominal cavity is one of the most dangerous complications(Jeans et al., 2007).Intense chronic foreign body reactions around the mesh prosthesis may produce meshoma /plugoma treatment of which becomes a new surgical challenge(Benedetti et al., 2005). The observed complication rate and postoperative dysfunction and high cost composite meshes have urged 
surgeons to look for new hernia repair techniques or to modify old ones. An example of such efforts is the Desarda's method, introduced in2001 and became a new surgical option for tissue-based inguinal hernia repair.

\section{Patients:}

This study was conducted at general surgery department of Qena university teaching hospital. The study period was one year, from December 2018 to December 2019. The study included 81 patients with inguinal hernia. Patients with huge inguino scrotal, recurrent, obstructed or strangulated hernia and those with mental or otherco-morbid conditions were excluded. Patients with weak or divided external oblique aponeurosis were also excluded.

\section{Methods:}

All of patients had been subjected to the following:

Detailed history, examination and investigations were done on all patients to assess fitness and to rule out comorbid conditions. Patients were also evaluated for any predisposing condition for inguinal hernia. Patients with obvious predisposing condition were managed accordingly before surgery was done. Desarda repair was done after taking informed consent from each patient. Patients were followed for 12 months post operatively, and complications were noted and rates calculated to assess the outcome.

\section{Statistical analysis:}

Data were entered into a computer using Epidata-Entry3.1 and then exported to the statistical package STATA 10 for analysis. Analysis was based on an intention-to-treat design. Mean pain score, day of return to normal gait and operative time were compared using Student's $t$ test. Comparison of complication rates was performed by $\chi 2$ (Chi-square) or Fisher's exact test. Bivariate and multivariate analysis using $\mathrm{t}$ test, $\chi 2$, analysis of variance (ANOVA) with Bonferroni tests and multiple regression analysis were done to evaluate the influence of baseline factors on the key outcomes. The power of the study was set at $80 \%$, confidence interval at $95 \%$ and a two-sided $\mathrm{P}<0.05$ was considered statistically significant. Accuracy of randomisation was analysed using the $\chi 2$ Contingency Table Test and evaluation of effectiveness of blinding was also analysed using percentage agreements.

\section{Results}

Our study included 126 patients with inguinal or inguinoscrotal hernia seen during the study period, 81 patients were eligible and included in the study. Forty five patients were excluded during the operation and the remaining 81 patients were randomly allocated into 2 groups. Three patients were lost during follow up period and were excluded from further analysis in the study. Finally, the analyzed patients were 40 patients in Desarda repair group and 41 patients in Lichtenstein group. Demographic data, age, gender, BMI, and comorbidities were comparable in both groups. No significant difference in the characters of hernia was detected in both groups. There was no recurrence in each study group during the 12 months follow up period. Groin pain after 6 months was reported by $4(5.6 \%)$ and $3(4.2 \%)$ 
patients from group I and II respectively $(\mathrm{P}=0.68)$; pain was classified as moderate pain (VAS 3-5.5). The majority of participants $(62 \%)$ had inguinal hernias on the right side. There were 69/81 (78\%) indirect hernias, with the majority being in Nyhus class IIIB $(40 \%)$. The distribution of baseline demographic and clinical characteristics was similar in the two intervention arms.

\section{Pain score and mean day of return to normal gait}

There was no significant statistical difference in mean pain scores at the four time points between the two intervention groups $(\mathrm{P}>0.05)$. However, a noticeable, but not statistically significant difference was observed on the 3rd POD, with lower mean pain scores among the non-mesh subjects. The difference of the mean day of return to normal gait between the groups was not statistically significant $(P=0.1722)$



Figure (1)pain score trend

The general trend showed an increase in pain score on the 3rd POD, followed by a marked decline in scores on the 7th day, with the pain score being nearly zero on the 14th day in the non-mesh group. One way analysis of pain score and treatment arm showed a noticeable difference in pain scores on the 3rd day. However, this was not statistically significant.

\section{Days to Return to Normal Gait}

The majority of participants in the study population returned to normal gait within 2-3 days. Four participants in the nonmesh group and three in the mesh group resumed normal gait $4 \mathrm{~h}$ after surgery. However, the lone participant in the mesh group who resumed normal gait on the 10th day had also developed a moderate scrotal haematoma on the 3rd POD, with pain scores of 7, 5 and 1 on the VAS on the 3rd, 7th and 14th POD, respectively.



Figure (2)Mean day of return to normal gait for the study population

Overall, the participants in the non-mesh group returned to normal gait earlier than those in the mesh group and the overall study population.

\section{Operative time}

Mesh repair took longer to accomplish, with a difference of 5.92 min compared to the non-mesh repair $(\mathrm{P}=0.0001)$. 


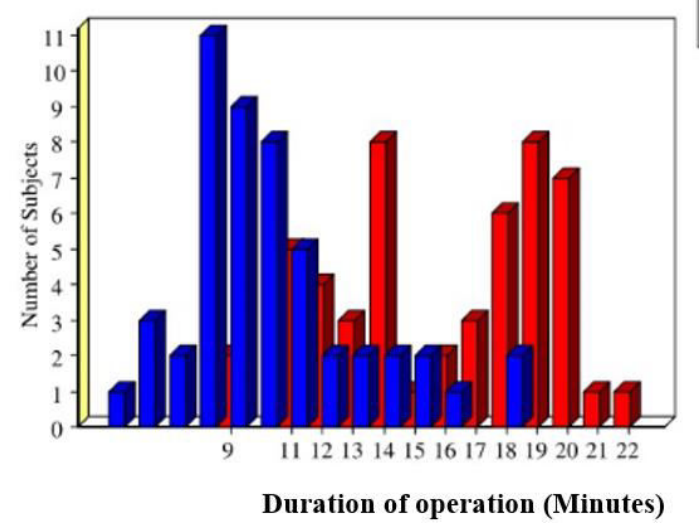

Figure (3) Operative time by method of repair

The great majority of participants in the mesh arm were operated within 13-20 min, whereas those in the non-mesh arm were operated within 8-11 min. Note that the operative time in this study refers to time taken to carry out only the actual repair of the posterior wall of the inguinal canal, not skin incision to skin closure time.

\section{Complications}

The proportion of patients experiencing any complications was similar between the mesh and non-mesh groups. there was no statistical difference between the two intervention arms $(\mathrm{P}=0.617)$.

Eight members developed moderate scrotal oedema in the examination, four occurring in mesh group and four in nonmesh group. Three scrotal haematoma were observed, two in the mesh group and one in the non-mesh group. All these complications are noticed in members with indirect, especially Nyhus class IIIB hernias. The patients with scrotal oedema and one patient with a small scrotal haematoma undergo conservative treatment. One male member, aged 54 in the mesh group, with a Nyhus class IIIB indirect hernia of 40 years duration, developed a small seroma. It is developed between the 4th and 6th postoperative day and had diminished by the 14th POD on conservative management. None of the members developed surgical site infection (wound sepsis). There was no statistical difference in the distribution of these complications.

\section{Discussion}

Inguinal hernia is a very common condition afflicting mankind. Newer techniques are developed as the complication rate of older ones become unacceptable. The use of mesh prosthesis for inguinal hernia repair has increased in popularity. The operation described by Lichtenstein is simple, safe and achieves all the goals of modern hernia surgery. The mesh prosthesis is expensive and not available all over the world especially in developing countries. Also when infected, the mesh has to be removed which further increases the morbidity and adds to the cost of the procedure.

Lichtenstein's technique and its modifications are widely practiced in the world but their complication rates and failures are more in the hands of nonconsultant staff. Mesh repair, plug repair, plug and mesh repair have all confused what is best and what to follow in the minds of such surgeons, who are not expert in hernia surgery.

The Desarda technique for inguinal hernia repair is a new tissue-based method. Despite the objections presented by some authors (Losanoff et al.,2001).application of the external oblique muscle aponeurosis in the form of an undetached strip (which makes the posterior wall of the inguinal canal stronger) has been established as a new concept in tissue based hernia repair. 
The technique is original, new, and satisfies the principles of "no tension" presented by Lichtenstein, and is different from the historical methods using the external oblique aponeurosis (McArthur, 1990).

Desarda's technique of inguinal hernia repair is easy to learn and does not require complicated dissection. As the steps in this surgery are fixed there is very less scope for modification by individual surgeon. This new technique of hernia repair does not need any costly mesh or laparoscopic instruments. This makes this repair highly cost effective. That is why many published

articles recently demonstrated an interest in the technique (Desarda, 2007; Situma, 2009). In our study, there were no statistically significant differences between the patients enrolled to the Desarda and Lichtenstein groups. The percentage of other early and late complications was comparable. The higher ratio of seromas after use of the Lichtenstein method can be explained by the influence of the synthetic mesh on surrounding tissues. This is consistent with other studies and the known influence of polypropylene on tissue (Grant, 2002; Horstmann et al., 2006).

There was no recurrence in either group. Similar findings were reported by Desarda MP (2006) (Desarda, 2006) on 860 patients over a follow up period of more than seven years. Mean VAS score on 1st post-operative day was 2.86 in Desarda's technique and 3.50 in Lichtenstein's technique. Similar study by Mitura K and Romanczuk M (2008) (Mitura; Romanczuk, 2008).Compared Desarda's and Lichtenstein's technique and reported mean VAS score on 1st post-operative day to be 3.3 and 3.8 in Desarda's and Lichtenstein's technique respectively. In our study the mean hospital stay was 2.58 days and 3.90 days in Desarda and Lichtenstein group respectively. Similar study by Mitura $\mathrm{K}$ and Romanczuk $\mathrm{M}$ (2008) reported that patients operated by Desarda's technique were discharged on 4th day and those operated by Lichtenstein's technique were discharged on 5th postoperative day.). The mean time to return to basic physical activity in was 7.04 and 11.30 days in Desarda's and Lichenstein's group respectively. Similarly study conducted by Desarda MP and Ghosh A (2006) (Desarda; Ghosh 2006)reported that the mean time to return to work in the Desarda's technique was 8.48 days while it was 12.46 days in the Lichtenstein's group.

\section{Conclusions:}

The Desarda's and Lichtenstein's methods of primary inguinal hernia repair do not differ in the means of procedure complexity, surgery time and complication profile. However, the return to basic physical activity was earlier in Desarda's repair. Desarda's no mesh repair is equally safe and more cost effective than Lichtenstein's repair. The technique may potentially increase the number of tissuebased methods available for treating inguinal hernias.

\section{Study Limitations}

- The methods of assessment of normal gait and pain are subject to participant and observer bias. An effort was made at every stage of the trial to blind the participant and the outcomes assessor. Any possible shortcomings pertaining to the methods of data collection were due mainly to the inherent problems of these methods. The outcomes 
assessor was specially trained prior to the start of the trial.

- With regard to postoperative complications, notably the absence of wound sepsis in this study, its generalisation to the general population is limited only to those patients with similar baseline characteristics, and who can afford prophylactic antibiotics.

- Since the follow up of the participants in this study was designed to be completed in 2 weeks, due to the limited time and funds available, some short-term complications that possibly occurred 2 weeks postoperatively were not documented. Two months would be a suitable period of follow-up to observe most of the delayed early complications of hernia repair.

\section{References:}

Amid PK. (2004). Lichtenstein tension-free hernioplasty: its inception, evolution, and principles. Hernia, 8:17.

\section{Bay-Nielsen M, Kehlet H, Strand L,} Malmstrøm J, Andersen F, Wara P. (2001). Quality assessment of 26,304 herniorrhaphies in Denmark: a prospective nationwide study. Lancet, 358:1124-1128.

Benedetti M, Albertario S, Niebel T, Bianchi C, Tinozzi F, Moglia $P$ et al., (2005). Intestinal perforation as a longterm complication of plug and mesh inguinal hernioplasty: case report. Hernia, 9:93-95.

\section{D'Amore L, Gossetti F, Vermeil V,NegroP. (2008). Long- termdiscomfort after plug and patch hernioplasty. Hernia, 12:445-446.}

Desarda MP. (2001). inguinal herniorrhaphy with an undetached strip of external oblique aponeurosis: a new approach used in 400 patients. Eur J Surg, 167:443-448.

Desarda MP. (2001). new method of inguinal hernia repair: a new solution. ANZ J Surg, 71:241-244.

Desarda MP.(2007). A new technique of inguinal hernia repair- neither similar to nor modification of Desarda's repair. J Indian Med Assoc, 105:654.

Desarda MP. (2008). No-mesh inguinal hernia repair with continuous absorbable sutures: a dream or reality? A study of 229 patients. Saudi J Gastroenterol, 14:122-127.

Fawole AS, Chaparala RPC, Ambrose NS.(2006). Fate of the inguinal hernia following removal of infected prosthetic mesh. Hernia, 10:58-61.

Genc V, Ensari C, Ergul Z, Kulacoglu H. (2010). A very late-onset deep infection after prosthetic inguinal hernia repair. Chirurgia (Bucur), 105:555-557.

Grant AM. (2002). Open mesh versus non-mesh repair of groin hernia: metaanalysis of randomised trials based on individual patient data [corrected]. Hernia, 6:130-136. 
Horstmann R, Hellwig M, Classen C, Röttgermann S, Palmes D.(2006). Impact polypropylene amount on functional outcome and quality of life after inguinal hernia repair by the TAPP procedure using pure, mixed, and titanium-coated meshes. World J Surg, 30:1742-1749.

Jeans S, Williams GL, Stephenson BM. (2007). Migration after open mesh plug inguinal hernioplasty: a review of the literature. Am Surg, 73:207-209.

Losanoff JE, Millis JM. (2006).Aponeurosis instead of prosthetic mesh for inguinal hernia repair: neither physiological nor new. Hernia 10:198-199 author reply 200102.

McArthur LL. (1901).Autoplastic suture in hernia and other diastases.JAMA. 37:1162-1165.

McRoy LL. (2010). Plugoma and the prolene hernia system. J Am CollSurg, 212:424 author reply 424-425.

Miller JP, Acar F, Kaimaktchiev VB, Gultekin S H, Burchiel K J. (2008). Pathology of ilioinguinal neuropathy produced by mesh entrapment: case report and literature review. Hernia, 12:213-216.

Mitura K,Romanczuk M. (2008).Comparison between two methods of inguinal hernia surgeryLichtenstein and Desarda. Pol MerkurLekarski, 24:392-395.

Ott V, Groebli Y, Schneider R. (2005). Late intestinal fistula formation after incisional hernia using intraperitoneal mesh. Hernia, 9:103104.

Primatesta P,Goldacre MJ. (1996).inguinal hernia repair: incidence of elective and emergency surgery, readmission and mortality. Int $\mathbf{J}$ Epidemiol, 25:835-839.

Ravitch MM,Hitzrot JM. (1960).The operations for inguinal hernia. I. Bassini, Halsted, Andrews, Ferguson Surgery, 48:439-466.

Scott NW, McCormack K, Graham P, Go PM, Ross, SJ, Grant AM. (2002). Open mesh versus non-mesh for repair of femoral and inguinal hernia. Cochrane Database Syst Rev, CD002197.

Situma SM. (2009). Comparison of Desarda versus modified Bassini inguinal Hernia repair: a randomized controlled trial. East Cent Afr J Surg, 14:70-76. 